\title{
Parathyroid hormone serum concentration kinetic profile in critically ill patients undergoing continuous renal replacement therapies: a prospective observational study
}

\author{
Aneta Czarnik', Ryszard Gawda ${ }^{2}$, Maciej Piwoda ${ }^{2}$, Maciej Marszalski², Maciej Molsa ${ }^{3}$, Marek Pietka ${ }^{3}$, \\ Marek Bolanowski ${ }^{\circledR 4}$, Tomasz Czarnik ${ }^{2}$ \\ ${ }^{1}$ Department of Endocrinology, Voivodeship Hospital in Opole, Opole, Poland \\ ${ }^{2}$ Department of Anaesthesiology, Intensive Care and Regional ECMO Centre, Institute of Medical Sciences, University of Opole, \\ Opole, Poland \\ ${ }^{3}$ Department of Anaesthesiology, Intensive Care and Regional ECMO Centre, Opole University Hospital, Opole, Poland \\ ${ }^{4}$ Department of Endocrinology, Diabetes, and Isotope Therapy, Wroclaw Medical University, Wroclaw, Poland
}

\begin{abstract}
Introduction: Elevated serum parathormone (PTH) levels have been observed in acute kidney injury and are related to calcium-phosphate metabolism disturbance, decreased renal production of 1,25 dihydroxyvitamin D3, impaired renal PTH excretion, and other renal-independent factors. There are no data regarding PTH concentration kinetics in critically ill patients undergoing continuous renal replacement therapies (CRRT) in an intensive care setting. The primary objective of this study was to investigate trends in PTH serum levels in critically ill patients with multiorgan failure undergoing CRRT, by performing periodic PTH measurements in the acute phase of critical illness. Material and methods: This was a single-centre, prospective, observational study conducted in an mixed, university-affiliated intensive care unit. Critically ill patients who fulfilled all of the following criteria were included: respiratory failure; circulatory failure; acute kidney injury treated by CRRT; and sequential organ failure assessment score (SOFA score) of 5 or more. Patients who met any of the following criteria were excluded: acute liver failure; hypercalcemia at admission (total calcium serum level $>10.6 \mathrm{mg} / \mathrm{dL}$; total ionized calcium plasma level $>1.35 \mathrm{mmol} / \mathrm{L}$ ); parathyroid gland disease; end-stage renal disease; patients undergoing therapeutic plasma exchange or extracorporeal membrane oxygenation procedures; aged under 18 years; pregnant; and life expectancy after admission to the intensive care unit anticipated to be less than 72 hours as assessed by the investigator.

Results: Thirty patients met the inclusion criteria. A statistically significant change in PTH over time was observed (Friedman ANOVA; $\mathrm{p}=0.0001)$. The post-hoc test showed a statistically significant decrease in PTH: measurements $5-8$ relative to measurement 1 , and measurements $4-8$ relative to measurement $2(\mathrm{p}<0.05)$. No significant correlations between 25 hydroxyvitamin D3 deficiency, age, diagnosis, SOFA score, and PTH levels were observed. A statistical test indicated that serum concentrations of PTH were significantly higher in the de novo sepsis group $(\mathrm{p}<0.05)$.

Conclusions: The PTH serum concentration decreases during the course of CRRT in the majority of patients. When the course of the disease starts to be complicated by sepsis, PTH serum levels then remain high. A probable reason for this is the existence of the inflammatory state triggered by sepsis. (Endokrynol Pol 2021; 72 (4): 329-335)
\end{abstract}

Key words: acute kidney injury; continuous renal replacement therapy; critical illness; intensive care; parathyroid hormone

\section{Introduction}

Parathyroid hormone (PTH) is secreted in the human body by the chief cells of the parathyroid glands. The bioactive, circulating form of PTH contains 84 amino acids and is formed from prohormone 115-amino acid sequence (preproPTH) by a cleavage process $[1,2]$. PTH is mainly responsible for the regulation of extracellular calcium and phosphate concentrations. PTH causes an increase in plasma calcium concentration and a decrease in plasma phosphate concentration [1, 2]. It mobilizes calcium and phosphate from the bone, decreases calcium excretion, and increases phosphate excretion by the kidneys, and finally, by stimulating the formation of 1,25 dihydroxyvitamin D3 in the kidneys, increases the intestinal absorption of calcium and phosphate.

Extracellular ionized calcium is the main regulator of PTH excretion from the parathyroid glands. Decreased levels of ionized calcium stimulate excretion, and increased levels inhibit it. The maximal stimulation of PTH excretion occurs when the plasma level of ionized calcium reaches $0.5 \mathrm{mmol} / \mathrm{L}$ or less. The maximal inhibition of PTH excretion occurs at an ionized calcium concentration of $2 \mathrm{mmol} / \mathrm{L}$ or more. The renally excreted, active form of vitamin $\mathrm{D}$, namely 1,25 dihydroxyvitamin D3, inhibits PTH gene transcription and parathyroid cell 
proliferation. There are many additional modulators of PTH secretion in the human body such as lithium, transforming growth factor alfa, prostaglandins, inorganic phosphate, and fibroblast growth factor 23 (FGF-23) $[1,2]$. FGF-23 inhibits renal phosphate reabsorption in the proximal renal tubules and reduces the circulatory level of 1,25 dihydroxyvitamin D3.

Besides the classic mechanisms, serum PTH concentrations can change in a non-serum calcium-dependent manner. PTH serum levels can increase in proportion to the severity of illness, infection, and inflammatory response [3]. It has been observed that significantly increased serum PTH levels correlate with the severity of critical illness, length of hospital stay, and mortality [4, 5]. PTH may also be treated as a marker of hypoperfusion, and elevated PTH serum levels reflect the risk of mortality in critically ill trauma patients [6]. However, it is unclear whether elevated PTH serum levels are isolated phenomena or reflect the severe perturbation of the 25 hydroxyvitamin D3-PTH-serum calcium axis in the course of critical illness $[7,8]$. Generally, the role of PTH in the pathophysiology of critical illness is under-researched.

Elevated serum PTH levels have also been observed in acute kidney injury. Elevated PTH serum levels are related to calcium-phosphate metabolism disturbance, decreased renal production of 1,25 dihydroxyvitamin D3, impaired or completely inhibited renal PTH excretion, and other renal-independent factors mentioned above [10-12]. Acute kidney injury is a commonly observed complication in intensive care, with mortality rates reaching $70 \%$ and the need for renal replacement therapy in up to $10 \%$ of cases [13]. Continuous renal replacement therapy (CRRT) is the standard of care in many intensive care units, especially when haemodynamic instability predominates the course of critical illness. Typical indications for CRRT in an intensive care setting are as follows: persistent/progressive acute kidney injury, volume overload, metabolic acidosis, electrolyte abnormalities, and uraemia [13]. In chronic kidney disease (end-stage renal disease) haemodialysis patients, changes in PTH serum levels depend on calcium status and the adsorptive properties of dialysis membranes [14]. Moreover, the composition of dialysis solutions influences changes of PTH serum concentrations during haemodialysis and haemodiafiltration procedures [15]. To date, there are no data regarding PTH kinetics in critically ill patients with multiorgan failure undergoing CRRT in an intensive care setting.

The primary objective of this study was to investigate trends in PTH serum levels in critically ill patients with multiorgan failure undergoing CRRT, by performing periodic serum PTH measurements in the acute phase of critical illness. The second objective was to assess whether factors not associated directly with acute kidney injury, like disease severity, admission diagnosis, vitamin D status, age, and course of critical illness, can influence the PTH kinetic profile. We hypothesize that PTH serum concentrations normally decrease during the course of CRRT in critically ill patients.

\section{Material and methods}

This was a prospective observational study conducted from May 2019 to October 2020 in a single 11-bed, mixed, university-affiliated intensive care unit (ICU). Written informed consent was obtained from the patients' relatives. The study was approved by the Regional Ethics Committee in Opole, Poland (protocol number: 278/2019; date of approval: 07/03/2019), registered before the recruitment of participants (clinicaltrials.gov; Identifier: NCT03873675) and carried out according to the principles of the Declaration of Helsinki. Consecutive patients undergoing CRRT were evaluated for participation in the study. Critically ill patients were included who fulfilled all of the following criteria: (1) respiratory failure; (2) circulatory failure; (3) acute kidney injury treated by continuous veno-venous hemodiafiltration (CVVHDF), which was started no later than 48 hours after admission to the ICU; and (4) a sequential organ failure assessment score (SOFA score) of five or more. We defined respiratory failure as a condition with clinical indications for invasive mechanical ventilation, and circulatory failure as a need for inotrope or/and vasopressor therapy. Indications for CRRT were based upon the KDIGO Clinical Practice Guidelines for Acute Kidney Injury document [16]. CVVHDF was performed using regional citrate anticoagulation, Prismaflex system and ST150 set (Prismaflex, Gambro Lundia AB, Lund, Sweden) with polyacrylonitrile membrane (AN69). Continuous intravenous ionized calcium supplementation was performed using an external infusion pump based on a dedicated citrate anticoagulation algorithm and kept within normal values during CRRT (hospital laboratory reference values: $1.15-1.33 \mathrm{mmol} / \mathrm{L}$ ). Phosphate serum levels were monitored and supplemented as needed during patients' stay in the intensive care unit (the standard of care in our institution) and also kept within normal values during CRRT (hospital laboratory reference values: $2.5-4.5 \mathrm{mg} / \mathrm{dL}$ ).

Patients who met any of the following criteria were excluded: acute liver failure; hypercalcaemia at admission (total calcium serum level > $10.6 \mathrm{mg} / \mathrm{dL}$; total ionized calcium plasma level > $1.35 \mathrm{mmol} / \mathrm{L}$ ); parathyroid gland disease; end-stage renal disease; patients undergoing therapeutic plasma exchange or extracorporeal membrane oxygenation (ECMO) procedures; aged under 18 years; pregnant; and those with life expectancy after admission to the intensive care unit anticipated to be less than 72 hours as assessed by the investigator.

The intact PTH was measured. Blood samples were taken from an arterial line or central venous line, or by direct peripheral venous or arterial puncture, and were collected in ethylenediamine tetra-acetic acid (EDTA) tubes. Blood samples were protected from exposure to light, transported to the hospital laboratory within 30 minutes, centrifuged at $3500 \mathrm{rpm}$ for 10 minutes, and processed by laboratory technicians. The PTH serum level was measured using an electrochemiluminescence ECLIA binding assay on Cobas e411 or Cobas 6000 immunoassay analysers (Roche Diagnostics $\mathrm{GmbH}$, Mannheim, Germany). The PTH reference values were set between 14.9 and $56.9 \mathrm{pg} / \mathrm{mL}$.

Consecutive patients admitted to the ICU were assessed in terms of study participation (inclusion and exclusion criteria) by the study investigator. In the majority of patients, the first PTH serum level was measured at the time of admission to the ICU. The first serum PTH level was measured before the initiation of CRRT. The next set of PTH serum levels was taken in 12-hour time intervals (twice daily, at 6 a.m. and 6 p.m.). The minimum necessary number of PTH measurements was six and the maximum was eight. The levels of 25 hydroxyvitamin D3, albumin and protein, coagulation 
tests, and bilirubin were measured at admission to the ICU. Ionized calcium and postfilter calcium were measured four times per day according to the CRRT protocol. Additional laboratory data such as blood count, serum creatinine, urea, phosphate, total calcium, and magnesium were measured once a day during the study period. All demographic data (date, name, hospital documentation number, sex, age, diagnosis at admission, comorbidities, admission SOFA score, and additional laboratory tests) were recorded in the hospital's electronic database. After the recruitment process, patient data were extracted from the electronic database, the patient's identification was blinded, and the data were transferred to a statistician for analysis.

\section{Statistical methods}

The quantitative variables were characterized by the arithmetic mean of standard deviation or median or max/min (range). The qualitative variables were presented using counts and percentages. In order to check whether a quantitative variable derived from a population with normal distribution, the W Shapiro-Wilk test was used. To prove the hypotheses on homogeneity of variances, the Levene (Brown-Forsythe) test was used. The statistical significance of differences between two groups was processed with Student's t-test or Mann-Whitney U test. The significance of difference between more than two groups was assessed with the F test (ANOVA) or Kruskal-Wallis test. In the case of statistically significant differences between two groups, post hoc tests were used (Tukey test for F or Dunn for Kruskal-Wallis). In the case of two paired variables, model Student's t-test or Wilcoxon signedrank test was used. The significance of difference between more than two variables in the paired variables model was checked by analysis of variance with repeated measurements or by the Friedman test. Chi-squared tests for independence were used for qualitative variables. In order to determine the dependence, strength and direction between variables, correlation analysis was used by determining the Pearson or Spearman's correlation coefficients. In all the calculations a statistical significance level of $p=0.05$ was used. The statistical analyses were performed using STATISTICA software v. 12.0 (StatSoft Inc., 2014).

\section{Results}

A total of 79 patients were evaluated for participation in the study. Forty-nine patients were excluded. The reasons for exclusion were as follows: no respiratory failure (9 patients), end-stage renal disease (6 patients), less than 6 PTH measurements performed (14 patients), CRRT started $>48$ hours after admission to the intensive care unit (8 patients), ECMO (4 patients), anticipated life expectancy after admission to the intensive care unit of less than 72 hours ( 4 patients), and others (4 patients). Finally, 30 patients met the inclusion criteria and were included in the study. The baseline demographics for the study group are shown in Table 1 and 2 . All patients fulfilled the KDIGO criteria of stage 3 acute kidney injury. The prescribed CVVHDF dose (effluent flow rate) was set between 30 and $42 \mathrm{~mL} / \mathrm{kg} / \mathrm{h}$. Other CRRT parameters were as follows: blood flow $120-160 \mathrm{~mL} / \mathrm{min}$; dialysate flow $1000-1500 \mathrm{~mL} / \mathrm{h}$; replacement fluid flow $600-1500 \mathrm{~mL} / \mathrm{h}$; and citrate supply $2.6-3.6 \mathrm{mmol} / \mathrm{L}$. The net fluid loss was set between 0 and $200 \mathrm{~mL} / \mathrm{h}$. In the majority of patients, the ratio of diffusion to convection was set between 1:1 and 1:0.5.
Table 1. Descriptive statistics (age, gender, BMI, serum concentrations of 25 hydroxyvitamin D3, and the SOFA score)

\begin{tabular}{lc}
\hline & Study group $(\mathbf{n}=\mathbf{3 0})$ \\
\hline Age (years) & $66.5(13.0)$ \\
\hline Mean (SD) & \\
\hline Gender & $12(40.0 \%)$ \\
\hline Female & $18(60.0 \%)$ \\
\hline Male & \\
\hline BMI $\left[\mathbf{k g} / \mathbf{m}^{2}\right]$ & $25.9(3.8)$ \\
\hline Mean $(\mathrm{SD})$ & \\
\hline $\mathbf{2 5}$ hydroxyvitamin D3 & $21(70.0 \%)$ \\
\hline$<12.5 \mathrm{ng} / \mathrm{mL}$ & \\
\hline SOFA & 11.0 \\
\hline Median & $6.0-16.0$ \\
\hline Range & \\
\hline
\end{tabular}

SD — standard deviation; BMI — body mass index; SOFA — sequential organ failure assessment score

Table 2. Descriptive statistics: diagnosis at admission

\begin{tabular}{lc}
\hline Diagnosis & Study group $(\mathbf{n}=\mathbf{3 0})$ \\
\hline Septic shock & $11(36.7 \%)$ \\
\hline Sudden cardiac arrest & $8(26.7 \%)$ \\
\hline Multiple organ dysfunction & $8(26.7 \%)$ \\
\hline Acute pancreatitis & $1(3.3 \%)$ \\
\hline Haemorrhagic shock & $1(3.3 \%)$ \\
\hline Intracranial bleeding & $1(3.3 \%)$ \\
\hline
\end{tabular}

The median serum concentration of PTH (first measurement) was $180.5 \mathrm{pg} / \mathrm{mL}(5.0-668.0)$, the $2^{\text {nd }}$ measurement was $166.5 \mathrm{pg} / \mathrm{mL}$ (15.0-347.0), the $3^{\text {rd }}$ measurement was $71.0 \mathrm{pg} / \mathrm{mL}(16.0-182.0)$, the $4^{\text {th }}$ measurement was $55.5 \mathrm{pg} / \mathrm{mL}(16.0-196.0)$, the $5^{\text {th }}$ measurement was $57.5 \mathrm{pg} / \mathrm{mL}(15.0-127.0)$, the $6^{\text {th }}$ measurement was $64.5 \mathrm{pg} / \mathrm{mL}(15.0-205.0)$, the $7^{\text {th }}$ measurement was 57.5 $\mathrm{pg} / \mathrm{mL}(15.0-296.0)$, and the $8^{\text {th }}$ measurement was 62.0 $\mathrm{pg} / \mathrm{mL}$ (17.0-199.0). A summary of the statistical analysis of PTH serum concentration is shown in Table 3, the change in average values of PTH in Figure 1, and the change in medians in Figure 2. The statistical test (Friedman ANOVA) showed a statistically significant change in PTH over time ( $p=0.0001)$. The post-hoc test showed a statistically significant decrease in PTH: measurements $5-8$ relative to measurement 1 , and measurements $4-8$ relative to measurement $2(\mathrm{p}<0.05)$. No statistically significant changes were found for the remaining comparisons $(\mathrm{p}>0.05)$.

Seventeen patients in the study group $(56.7 \%)$ were discharged from the ICU, and 13 patients $(43.3 \%)$ 
Table 3. Summary statistical analysis of parathormone (PTH) serum concentrations PTH reference values: 14.9-56.9 [pg/mL]

\begin{tabular}{lc}
\hline & Study group $(\mathbf{n}=\mathbf{3 0})$ \\
\hline PTH - 1 & 180.5 \\
\hline Median & $5.0-668.0$ \\
\hline Range & 166.5 \\
\hline PTH - 2 & $15.0-347.0$ \\
\hline Range & \\
\hline PTH - 3 & 71.0 \\
\hline Median & $16.0-182.0$ \\
\hline Range & 55.5 \\
\hline PTH - 4 & $16.0-196.0$ \\
\hline Median & 57.5 \\
\hline Range & $15.0-127.0$ \\
\hline PTH - 5 & \\
\hline Median & 64.5 \\
\hline Range & $15.0-205.0$ \\
\hline PTH - 6 & $15.0-296.0$ \\
\hline Median & \\
\hline Range & \\
\hline PTH - 7 & \\
\hline Median & \\
\hline Range & \\
\hline PTH - 8 & \\
\hline Median & \\
\hline Range & \\
\hline & \\
\hline
\end{tabular}

died. No significant correlations between outcome and PTH values were observed. A total of 21 patients (70\%) had severe 25 hydroxyvitamin D3 deficiency at admission to the ICU (25 hydroxyvitamin D3 serum level $<12.5 \mathrm{ng} / \mathrm{mL}$ ). No significant correlations between 25 hydroxyvitamin D3 deficiency and PTH levels were observed. Also, no significant correlations between age, admission diagnosis, SOFA score, and PTH values were found.

In 10 patients $(33.3 \%)$, de novo sepsis or septic shock was diagnosed during the measurement period based on Surviving Sepsis Campaign criteria [17]. Additionally, we noted elevated serum C-reactive protein (CRP) and procalcitonin levels in these patients (CRP $47-728 \mathrm{mg} / \mathrm{L}$; procalcitonin $0.6-400 \mathrm{ng} / \mathrm{mL}$ ). Different relations were observed in patients who became septic and patients who remained non-septic during the measurement period. A statistical test indicated that serum concentrations of PTH: 3-8 measurements were significantly higher in the de novo sepsis group

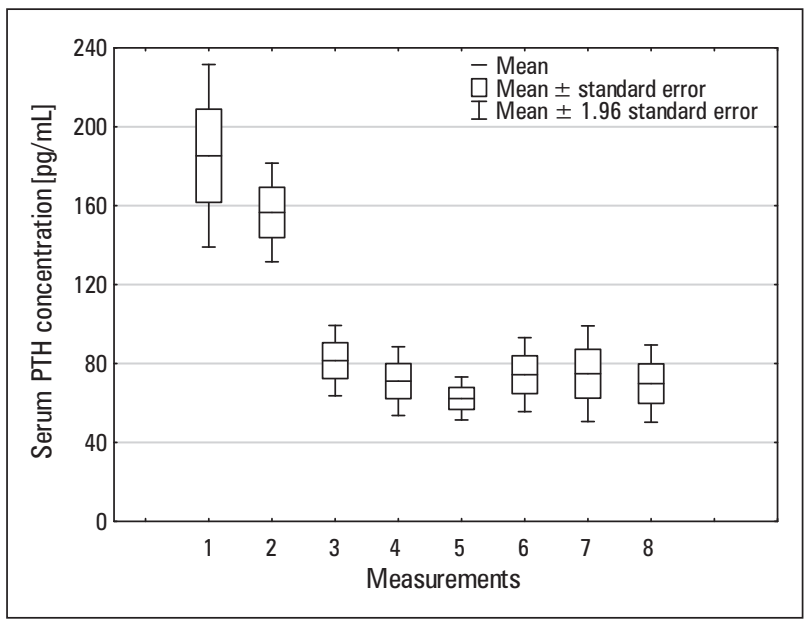

Figure 1. The change in parathormone (PTH) serum levels: average values. PTH reference values: $14.9-56.9$ [pg/mL]

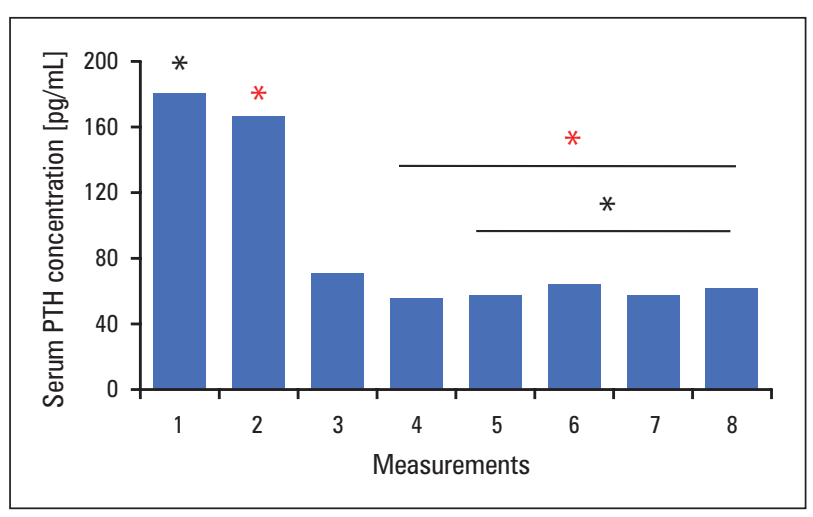

Figure 2. The change in parathormone (PTH) serum levels; medians $\left({ }^{*} * \mathrm{p}<0.05\right)$; PTH reference values: $14.9-56.9$ $[\mathrm{pg} / \mathrm{mL}]$

$(p<0.05)$ compared to the non-sepsis group. There were no significant differences between measurements of serum PTH concentrations in the de novo sepsis group $(p=0.7045)$. The statistical test (Friedman ANOVA) showed a statistically significant change in PTH serum levels over time in the non-sepsis group $(p=0.0001)$. The post-hoc test showed a statistically significant decrease in PTH serum concentrations: measurements 4-8 relative to measurement 1 and 2 $(p<0.05)$. No statistically significant changes were found for the remaining comparisons of measurements $(p>0.05)$. Comparative analysis of mean PTH serum concentrations in the de novo sepsis and non-sepsis groups are shown in Figure 3 and Table 4.

\section{Discussion}

We found a general trend in serum PTH concentrations in critically ill patients with multi-organ failure 


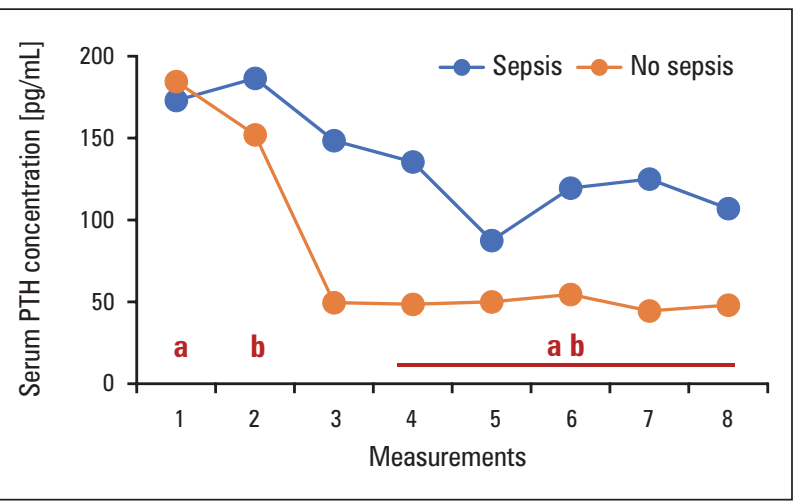

Figure 3. Comparative analysis of mean values of plasma concentration of parathormone $(\mathrm{PTH})$ in sepsis and nonsepsis groups ( $\left.{ }^{\mathrm{a}, \mathrm{b}} \mathrm{p}<0.05\right)$; PTH reference values: $14.9-56.9$ $[\mathrm{pg} / \mathrm{mL}]$

undergoing CRRT. In the majority of patients (25; $83.3 \%$ ), we noted significantly elevated serum PTH levels before starting the renal replacement procedure. This can mainly be attributed to the acute kidney injury and inhibited renal PTH elimination. The subsequent and gradual decrease in serum PTH concentrations in the course of CRRT can be explained by the washout process on the haemofilter. PTH has a molecular mass of 9500 daltons (Da). In the CVVHDF mode of CRRT used in all patients included in the study, there were two mechanisms responsible for the elimination process of particles, namely diffusion and convection. In the majority of our patients the ratio of diffusion to convection was set between 1:1 and 1:0.5. During the diffusion process, only small molecules can be removed. However, in the case of convection molecules with a greater molecular mass of up to 30,000 Da can be easily eliminated [18]. We assume that the decrease in PTH serum concentration in the majority of our patients was solely attributable to the convection process. Additionally, the PTH elimination process could be related to the adsorptive properties of the AN69 membrane. We assume that the accumulation of PTH in acute kidney injury before the beginning of CRRT is proportional to the period of disrupted kidney function, and inversely

Table 4. Comparative analysis of mean values of parathormone (PTH) serum concentrations in sepsis and non-sepsis groups PTH reference values: $14.9-56.9[\mathrm{pg} / \mathrm{mL}]$

\begin{tabular}{|c|c|c|c|}
\hline & Sepsis $(n=10)$ & Non-sepsis $(n=20)$ & p value \\
\hline PTH -1 & & & 0.5526 \\
\hline Median & 173.0 & 184.5 & \\
\hline Range & $20.0-306.0$ & $5.0-668.0$ & \\
\hline PTH -2 & & & 0.5500 \\
\hline Median & 186.5 & 152.0 & \\
\hline Range & $86.0-229.0$ & $15.0-347.0$ & \\
\hline PTH -3 & & & 0.0002 \\
\hline Median & 148.5 & 49.5 & \\
\hline Range & $28.0-182.0$ & $16.0-139.0$ & \\
\hline PTH -4 & & & 0.0019 \\
\hline Median & 135.5 & 48.5 & \\
\hline Range & $43.0-196.0$ & $16.0-88.0$ & \\
\hline PTH -5 & & & 0.0164 \\
\hline Median & 87.5 & 50.0 & \\
\hline Range & $30.0-127.0$ & $15.0-100.0$ & \\
\hline PTH - 6 & & & 0.0107 \\
\hline Median & 119.5 & 54.5 & \\
\hline Range & $24.0-189.0$ & $15.0-205.0$ & \\
\hline РTH -7 & & & 0.0008 \\
\hline Median & 125.0 & 44.5 & \\
\hline Range & $58.0-296.0$ & $15.0-127.0$ & \\
\hline PTH - 8 & & & 0.0070 \\
\hline Median & 107.0 & 48.0 & \\
\hline Range & $47.0-199.0$ & $17.0-83.0$ & \\
\hline
\end{tabular}


proportional to the degree of residual kidney function. In 10 patients $(33.3 \%)$ significantly elevated serum PTH levels were observed, which remained relatively high during subsequent measurements. The PTH washout during CRRT was preserved in septic patients, but the PTH production and excretion process was probably stimulated by inflammation. We can assume that trends in PTH serum concentrations can be used as another marker of inflammation. Further studies are needed to explore this phenomenon.

In a prospective observational cohort study, Greulich et al. found that patients with sepsis have high levels of PTH and lower blood levels of 25 hydroxyvitamin D3. The authors linked this phenomenon to a susceptibility to developing sepsis [19]. Severe 25 hydroxyvitamin D3 deficiency is common in critically ill patients. The serum concentrations of 25 hydroxyvitamin D3 are low and stable during the therapeutic process in the ICU, also in patients undergoing CRRT $[8,20]$. We assume that sharp rises in PTH serum concentrations are independent of vitamin D status and the vitamin D-PTH-calcium axis in the human body, but instead contribute to the inflammatory cascade during sepsis. Carlstedt et al. observed that elevated levels of serum PTH are common in critically ill patients and the extent of the increase is related to the severity of disease and mortality. This phenomenon was observed in a broad mixture of ICU patients and especially in two specific disease groups, namely septic patients and those subjected to major surgery. The authors linked this with a systemic inflammatory response but concluded that the direct influence of inflammatory mediators on the calcium responsiveness of the PTH release is unknown. The authors also hypothesized that catecholamines could stimulate PTH secretion because beta-adrenergic receptors are present on parathyroid cell surfaces [21]. In a prospective observational study, Yang et al. found that elevated PTH serum levels in post cardiac surgery patients with a clinical diagnosis of acute kidney injury were marginally associated with poor outcomes [22]. In a prospective cross-sectional study, Ardehali et al. observed that in neurosurgical ICU patients with increasing PTH serum levels, the risk of stay in the ICU increased by $2 \%$. The authors confirmed that PTH could be regarded as a marker of inflammation and disease severity [23]. In a prospective observational study, Fligor et al. highlighted the potential utility of PTH as a marker of hypoperfusion in trauma shock patients. The authors concluded that sequentially estimated serum PTH levels could be a useful index of effective resuscitation in shock [6].Based on the results of our study and the review of the literature, we hypothesize that the most probable reason for prolonged elevated serum PTH concentrations in critically ill patients is the inflammatory state in the course of critical illness. We do not know if there is a relationship between the extent of PTH serum elevation and the intensity of sepsis; hence, further studies are merited. Initially elevated serum PTH levels are common in multiorgan failure with the coexistence of acute kidney injury; however, CRRT tends to normalize these levels. When sepsis and inflammation begin to predominate, the decline of previously elevated serum PTH levels is impaired.

Our study has several limitations. The first is the small number of patients included. However, this is the first study aimed at estimating the PTH serum concentration kinetic profile during CRRT. A larger cohort would be more accurate to evaluate above-mentioned relations. The second limitation is that we did not measure the broad spectrum of inflammatory markers in our study. The diagnosis of sepsis was based solely on clinical symptoms as well as commonly used laboratory markers such as C-reactive protein, procalcitonin, and leukocytosis. PTH stimulates interleukin- 6 production by osteoblasts and the liver, and this specific marker of inflammation would be more effective in the evaluation of the intensity of inflammation [24, 25]. The third is that we did not measure PTH concentration in the effluent, which would potentially show the range of $\mathrm{PTH}$ washout during CRRT. However, the PTH elimination process is probably related to the adsorptive properties of AN69 membrane as well. Finally, the study population was rather heterogeneous. However, our patients represent a typical, broad intensive care milieu, and substantially reflect typical intensive care scenarios.

\section{Conclusions}

Critically ill patients with acute kidney injury undergoing continuous renal replacement therapies initially have elevated serum PTH concentrations. We found that the PTH serum concentration decreases rapidly during the course of CRRT in the majority of patients. When the course of the disease starts to be complicated by sepsis, PTH serum levels then remain high. The most probable reason for this phenomenon could be the existence of the inflammatory state triggered by sepsis.

\section{Funding}

This research did not receive any specific grant from funding agencies in the public, commercial, or not-for-profit sectors.

\section{Conflicts of interest}

None declared.

\section{Clinical trial registration}

Clinicaltrials.gov; Identifier: NCT03873675. 


\section{Authors' contribution}

Study design - A.C., T.C., M.B.; conduct of the trial and data collection: A.C., T.C, R.G., M.Piw., M.Mar., M.Mol., M.Pie.; data analysis and manuscript preparation - A.C., T.C., R.G., M.B.; contribution to the final version of the manuscript: A.C., T.C., R.G., M.B., M.Piw., M.Mar., M.Mol., M.Pie.; general responsibility and quality control - A.C., T.C., M.B.

\section{References}

1. Goltzman D. Physiology of Parathyroid Hormone. Endocrinol Metab Clin North Am. 2018; 47(4): 743-758, doi: 10.1016/j.ecl.2018.07.003, indexed in Pubmed: 30390810.

2. Goltzman D, Mannstadt M, Marcocci C. Physiology of the Calcium-Parathyroid Hormone-Vitamin D Axis. Front Horm Res. 2018; 50: 1-13, doi: 10.1159/000486060, indexed in Pubmed: 29597231.

3. Lind L, Carlstedt F, Rastad J, et al. Hypocalcemia and parathyroid hormone secretion in critically ill patients. Crit Care Med. 2000; 28(1):93-99, doi: 10.1097/00003246-200001000-00015, indexed in Pubmed: 10667505.

4. Carlstedt F, Lind L, Wide L, et al. Serum levels of parathyroid hormone are related to the mortality and severity of illness in patients in the emergency department. Eur J Clin Invest. 1997; 27(12): 977-981, doi: 10 1046/j.1365-2362.1997.2310778.x, indexed in Pubmed: 9466123.

5. Burchard KW, Gann DS, Colliton J, et al. Ionized calcium, parathormone, and mortality in critically ill surgical patients. Ann Surg. 1990; 212(4): 543-9; discussion 549, doi: 10.1097/00000658-199010000-00016, indexed in Pubmed: 2222019.

6. Fligor SC, Love KM, Collier BR, et al. Parathyroid hormone as a marker for hypoperfusion in trauma: A prospective observational study. J Trauma Acute Care Surg. 2017; 83(6): 1142-1147, doi: 10.1097/TA.0000000000001656, indexed in Pubmed: 28700412.

7. Nair P, Lee P, Reynolds C, et al. Significant perturbation of vitamin D-parathyroid-calcium axis and adverse clinical outcomes in critically ill patients. Intensive Care Med. 2013; 39(2): 267-274 doi: 10.1007/s00134-012-2713-y, indexed in Pubmed: 23064465.

8. Czarnik T, Czarnik A, Gawda R, et al. Vitamin D kinetics in the acute phase of critical illness: A prospective observational study. J Crit Care. 2018; 43: 294-299, doi: 10.1016/j.jcrc.2017.09.179, indexed in Pubmed: 28968524.

9. Hu J, Luo Z, Zhao X, et al. Changes in the calcium-parathyroid hormone-vitamin $\mathrm{d}$ axis and prognosis for critically ill patients: a prospective observational study. PLoS One. 2013; 8(9): e75441, doi: 10.1371/journal pone.0075441, indexed in Pubmed: 24073266.

10. Pietrek J, Kokot F, Kuska J. Serum 25-hydroxyvitamin D and parathyroid hormone in patients with acute renal failure. Kidney Int. 1978; 13(2): 178-185, doi: 10.1038/ki.1978.25, indexed in Pubmed: 713278.

11. Kokot F, Mleczko Z, Pazera A. Parathyroid hormone, prolactin, and function of the pituitary-gonadal axis in male patients with acute renal failure. Kidney Int. 1982; 21(1): 84-89, doi: 10.1038/ki.1982.12, indexed in Pubmed: 6804685.
12. Fuss M, Bagon J, Dupont E, et al. Parathyroid Hormone and Calcium Blood Levels in Acute Renal Failure. Nephron. 2008; 20(4): 196-202, doi: 10.1159/000181222.

13. Tandukar S, Palevsky PM. Continuous Renal Replacement Therapy: Who, When, Why, and How. Chest. 2019; 155(3): 626-638, doi: 10.1016/j. chest.2018.09.004, indexed in Pubmed: 30266628.

14. De Francisco AL, Amado JA, Prieto M, et al. Dialysis membranes and PTH changes during hemodialysis in patients with secondary hyperparathyroidism. Nephron. 1994; 66(4): 442-446, doi: 10.1159/000187861, indexed in Pubmed: 8015649.

15. Šafránek R, Moučka P, Vávrová J, et al. Changes of serum calcium, magnesium and parathyroid hormone induced by hemodialysis with citrate-enriched dialysis solution. Kidney Blood Press Res. 2015; 40(1): 13-21, doi: 10.1159/000368478, indexed in Pubmed: 25661822

16. Kidney Disease: Improving Global Outcomes (KDIGO) Acute Kidney Injury Work Group. KDIGO Clinical Practice Guideline for Acute Kidney Injury. Kidney Int. 2012; 2012(Suppl): 1-138.

17. Rhodes A, Evans LE, Alhazzani W, et al. Surviving Sepsis Campaign: International Guidelines for Management of Sepsis and Septic Shock: 2016 Crit Care Med. 2017; 45(3): 486-552, doi: 10.1097/CCM.0000000000002255, indexed in Pubmed: 28098591.

18. Honore PM, Mugisha A, Kugener L, et al. Who may benefit most from future vitamin D intervention trials: do not forget patients on continuous renal replacement therapy. Crit Care. 2020; 24(1): 180 doi: 10.1186/s13054-020-02910-w, indexed in Pubmed: 32345332.

19. Greulich T, Regner W, Branscheidt M, et al. Altered blood levels of vitamin $\mathrm{D}$, cathelicidin and parathyroid hormone in patients with sepsis-a pilot study. Anaesth Intensive Care. 2017; 45(1): 36-45, doi: 10 1177/0310057X1704500106, indexed in Pubmed: 28072933.

20. Czarnik T, Czarnik A, Gawda R, et al. Vitamin D serum levels in multiorgan failure critically ill patients undergoing continuous renal replacement therapies. Anaesthesiol Intensive Ther. 2020; 52(5): 359-365, doi: 10.5114/ait.2020.101008, indexed in Pubmed: 33242935.

21. Carlstedt F, Lind L, Rastad J, et al. Parathyroid hormone and ionized calcium levels are related to the severity of illness and survival in critically ill patients. Eur J Clin Invest. 1998; 28(11): 898-903, doi: 10.1046/j. 1365-2362.1998.00391.x, indexed in Pubmed: 9824432.

22. Yang $T$, Wang $W$, Tang $X$, et al. Association between minera and bone disorder in patients with acute kidney injury following cardiac surgery and adverse outcomes. BMC Nephrol. 2019; 20(1): 369, doi: 10.1186/s12882-019-1572-y, indexed in Pubmed 31615432.

23. Ardehali SH, Dehghan S, Baghestani AR, et al. Association of admission serum levels of vitamin D, calcium, Phosphate, magnesium and parathormone with clinical outcomes in neurosurgical ICU patients. Sci Rep. 2018; 8(1): 2965, doi: 10.1038/s41598-018-21177-4, indexed in Pubmed: 29445220.

24. Cheng SP Liu CL, Liu TP et al Association between parathyroid hormone levels and inflammatory markers among US adults. Mediators Inflamm. 2014; 2014: 709024, doi: 10.1155/2014/709024, indexed in Pubmed: 24782595.

25. Mitnick MA, Grey A, Masiukiewicz U, et al. Parathyroid hormone induces hepatic production of bioactive interleukin- 6 and its soluble receptor. Am J Physiol Endocrinol Metab. 2001; 280(3): E405-E412, doi: 10.1152/ajpendo.2001.280.3.E405, indexed in Pubmed: 11171594. 